\title{
Energy drives the dynamic localization of cyanobacterial nitrogen regulators during diurnal cycles
}

Javier Espinosa $^{1}{ }^{(\mathbb{0}}$, José I. Labella $^{1}$, Raquel Cantos and Asunción Contreras ${ }^{2 *}$

Departamento de Fisiología, Genética y Microbiología, Universidad de Alicante, Alicante, Spain.

${ }^{1}$ These authors contributed equally to this work.

${ }^{2}$ Lead contact.

*Correspondence: Asunción Contreras contrera@ua.es

Universidad de Alicante, Ctra. San Vicente del Raspeig s/n 03690 Spain. +34965909348

Running Title: Energy signalling during diurnal cycles

Significance Statement

Phototrophic organisms must adapt their metabolic processes to the challenges imposed by the succession of days and nights. PII proteins, found in all three domains of life as integrators of signals of the nitrogen and carbon balance as well as of energy signals, function by forming proteinprotein interactions with a variety of diverse targets. PipX, also mediating its function by proteinprotein interactions, is unique to cyanobacteria. We show here that PII and PipX proteins display distinct localization patterns during diurnal cycles and that at dark periods they co-localize into the same foci at the periphery and poles of the cells. Localization of PII-PipX into foci is driven by environmental signals modulating cellular ATP/ADP ratio in a circadian-independent manner. This works contributes to understand the role of the nitrogen regulators PII and PipX as integrators and transducers of key environmental signals in cyanobacteria.

This article has been accepted for publication and undergone full peer review but has not been through the copyediting, typesetting, pagination and proofreading process which may lead to differences between this version and the Version of Record. Please cite this article as an 'Accepted Article', doi: 10.1111/1462-2920.14071 


\section{SUMMARY}

Cyanobacteria, phototrophic organisms performing oxygenic photosynthesis, must adapt their metabolic processes to the challenges imposed by the succession of days and nights. Two conserved cyanobacterial proteins, PII and PipX, function as hubs of the nitrogen interaction network, forming complexes with a variety of diverse targets. While PII proteins are found in all three domains of life as integrators of signals of the nitrogen and carbon balance, PipX proteins are unique to cyanobacteria, where they provide a mechanistic link between PII signalling and the control of gene expression by the global nitrogen regulator NtcA. Here we demonstrate that PII and PipX display distinct localization patterns during diurnal cycles, co-localizing into the same foci at the periphery and poles of the cells during dark periods, a circadian-independent process requiring a low ATP/ADP ratio. Genetic, cellular biology and biochemical approaches used here provide new insights into the nitrogen regulatory network, calling attention to the roles of PII as energy sensors and its interactions with PipX in the context of essential signalling pathways. This study expands the contribution of the nitrogen regulators PII and PipX to integrate and transduce key environmental signals that allow cyanobacteria to thrive in our planet. 


\section{INTRODUCTION}

Diurnal cycles impose drastic changes to living organisms, which must adapt their metabolism in response to the succession of days and nights. The affected processes are usually synchronized with the day and night cycles through diurnal rhythms or through circadian rhythms, which are self-sustained biological rhythms of 24 hours. The need to ensure efficient diurnal regulation of metabolic processes is particularly important in cyanobacteria, phototrophic organisms that convert solar irradiance into chemical energy. They are the simplest organisms known to have a circadian clock, extensively studied in the model cyanobacterium Synechococcus elongatus PCC7942 (hereafter S. elongatus) (Shultzaberger et al., 2015), where it comprises a core oscillator which relay timing information to the output pathway, generating genome-wide transcriptional oscillations (Liu et al., 1995). Cross-talk with other two-component regulators allows integration of additional environmental signals (Markson et al., 2013; Espinosa et al., 2015; Boyd et al., 2016). In S. elongatus, diurnal fluctuations appear to be less pronounced at the protein level than at the transcript levels (Guerreiro et al., 2014). However, important differences between light and dark conditions affect mega Dalton protein assemblies (Guerreiro et al., 2016), supporting the importance of protein complexes and protein interaction networks in cyclic regulation of cellular processes.

The nitrogen interaction network of $S$. elongatus, the paradigm for nitrogen regulation in cyanobacteria, responds to signals of energy and Carbon/Nitrogen $(\mathrm{C} / \mathrm{N})$ balance (Huergo et al., 2013). The hubs of the network are PII, one of the most conserved signalling proteins (Chellamuthu et al., 2013; Merrick, 2014; Forchhammer and Luddecke, 2016), and the cyanobacterial factor PipX, identified by its ability to bind to PII (Burillo et al., 2004; Espinosa et al., 2006). PII and PipX mediate protein-protein interactions with regulatory targets that include transcriptional regulators, enzymes and transporters involved in nitrogen and/or carbon assimilation.

PII perceive metabolic information by competitive binding of ATP or ADP and by synergistic binding of ATP and 2-oxoglutarate (2-OG). The PII trimer has three binding sites for ATP/ADP (in 
some species AMP) and 2-OG (Fokina et al., 2010; Palanca et al., 2014). PII binds to N-acetyl-Lglutamate kinase (NAGK), stimulating its activity and promoting nitrogen storage as arginine in cyanobacteria and plants (Burillo et al., 2004; Heinrich et al., 2004; Llacer et al., 2007), and to the biotin carboxyl carrier protein (BCCP) of acetyl-CoA carboxylase (ACCase), inhibiting its activity to control acetyl-CoA levels in organisms encoding PII (Feria Bourrellier et al., 2010; Gerhardt et al., 2015; Hauf et al., 2016). PII-dependent inhibition of nitrate transport is known to occur after addition of ammonium to nitrate-containing cultures (Lee et al., 1998), a function that, although poorly characterized at the molecular level, depends on the NrtC subunit of the nitrate transporter (NRT) (Kobayashi et al., 1997).

When abundant, 2-OG binds to MgATP-complexed PII, triggering conformational changes that prevent the interaction of PII with either NAGK or PipX (Espinosa et al., 2006; Llacer et al., 2010; Zeth et al., 2014). In the absence of 2-OG, only the ATP/ADP ratio and concentration of ADP directs the competitive interaction of PII with those targets in vitro (Luddecke and Forchhammer, 2015). PipX increases the affinity of PII for ADP, and, conversely, the interaction between PII and PipX is highly sensitive to fluctuations in the ATP/ADP ratio (Zeth et al., 2014). These interactions provide a mechanistic link between PII signalling and gene expression. PipX uses the same surface to bind to either 2-OG-bound NtcA, stimulating DNA binding and transcriptional activity, or to 2OG-free PII to form PII-PipX complexes (Tanigawa et al., 2002; Vazquez-Bermudez et al., 2002; Llacer et al., 2010; Zhao et al., 2010). PII-PipX complexes interact with the transcriptional regulator PlmA, presumably to decrease transcriptional activity (Labella et al., 2016).

Fig 1 schematically illustrates the nitrogen interaction network and the position of PII and PipX as regulatory hubs, with information on the relative abundance of the protein components and the intracellular signal molecules determining protein-protein interactions (2-OG levels and the ATP/ADP ratio). Protein components included in the illustration are the three target proteins whose interactions with the hubs have been characterized to certain extent at the molecular level (NAGK, 
NtcA, BCCP). On the basis of genetic evidence we also included interactions of PII and PII-PipX with NrtC and PlmA, respectively, which should be favored by relatively low 2-OG levels and low ATP/ADP ratios. NrtC activity is inhibited by PII after addition of ammonium or transfer of cultures to darkness, while PlmA interactions require already formed PII-PipX complexes. Except for PipX, where concentric circles represent monomers (outer circle) and trimers (inner circles), the known or predicted quaternary structure of the proteins involved served to illustrate protein abundance.

PII and PipX are likely to play unknown roles in adaptation to environmental situations faced by photosynthesizing cyanobacteria. In particular, genetic analyses are consistent with a greater complexity of the protein-protein interaction network involving PII and PipX regulators and thus suggest that they may be integrating multiple signalling pathways. Particularly interesting is the phenomenon described as the toxicity of PipX in the absence of PII, which implicate both proteins in the regulation of essential processes in S. elongatus (Espinosa et al., 2009; Espinosa et al., 2010; Laichoubi et al., 2012; Chang et al., 2013), an implication also supported by in silico (Laichoubi et al., 2011) and transcriptomic analyses (Espinosa et al., 2014). However, the molecular bases of the relevant signalling pathway(s) inferred from these studies remain to be elucidated.

We show here that PII and PipX proteins display distinct localization patterns during diurnal cycles and that at dark periods they co-localize into the same foci at the periphery and poles of the cells. Localization of PII-PipX into foci is driven by a low ATP/ADP ratio in a circadianindependent manner. In addition, genetic analyses provide new insights into the multi-functionality of both PII and PipX, underscoring the role of PII as a negative regulator of PipX activities.

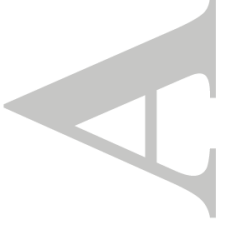




\section{RESULTS AND DISCUSSION}

\section{PII-YFP and PipX-GFP co-localize into foci during the dark period of diurnal cycles in a circadian independent manner}

To get insights into the localization of PII, PipX and their protein complexes in S. elongatus under different environmental conditions, we engineered compatible PipX-GFP (GFPmut2) and PIIYFP (EYFP) fusion proteins, each of which could be analysed distinctly in WT or mutant backgrounds (Fig S1A and B). To minimise artefacts, the fusion proteins were expressed from their corresponding promoters. Appropriated expression of the fusion proteins was subsequently verified by Western blots (Fig S1C).

To visualise the localization of PipX-GFP and PII-YFP by confocal microscopy, cultures of S. elongatus expressing both proteins were grown with nitrate under continuous light or under alternating (12:12) light-dark (LD) conditions. Cells grown under continuous light or during the light period of LD conditions showed homogeneous cytoplasmic distribution of the fluorescence (Fig 2A, Light-labelled panels). However, fluorescent foci corresponding to both PipX-GFP and PII-YFP were observed during the dark period of LD cultures (Fig 2A, Dark-labelled panels). To minimize bleed-through of the GFP and YFP signals, precise spectral settings were designed (Fig S2).

Importantly, both types of fluorescent foci were always observed at exactly the same positions in individual cells, indicating that the two proteins co-localize into the same foci. After transfer from light to dark, fluorescent foci were observed irrespective of the nitrogen regime of cultures. Foci were formed when nitrogen starvation was applied simultaneously to darkness, but not when cultures have been previously nitrogen deprived for several hours (Fig 2A and Fig S3), in line with the light dependence of nitrogen assimilation processes in S. elongatus (Flores, 1994).

S. elongatus cells co-expressing PipX-GFP and PII-YFP fusion proteins were subjected to diurnal cycles and the number of foci determined every 2 hours over a 28 hours period (Fig 2B). 
After 4 hours in the dark, around $60 \%$ of the cells had foci, whose numbers still increased slowly during the rest of the dark period. Disappearance after the subsequent light period was relatively fast, with almost no foci detectable after 4 hours of light. Their localization was mainly peripheral, with preference for the cell poles (Fig 2A, heatmaps, Dark). The number of detectable PipX-GFP or PIIYFP foci per cell was very similar, varying between 0 and 4 (for simplicity, heatmaps and histograms shown in Fig 2A correspond to PipX-GFP foci).

Enhanced polar localization at night of components of the clock oscillator has been reported in S. elongatus (Cohen et al., 2014). In particular, the discrete YFP-KaiC foci observed near single poles of cells are reminiscent of the PipX-GFP/PII-YFP foci observed here. To compare the localization patterns of fluorescent KaiC under the same experimental conditions, we engineered $S$. elongatus cells expressing ZsG-KaiC (ZsGreen1) either by itself or in the presence of PII-YFP. As shown in Fig 2A, differences between PipX-GFP/PII-YFP and ZsG-KaiC foci were observed in both number and localization. About $80 \%$ of the cells exhibited ZsG-KaiC foci, typically one focus that showed an almost exclusive polar localization, thus in full agreement with previously reported data for YFP-KaiC (Cohen et al., 2014). Importantly, ZsG-KaiC and PII-YFP foci did not co-localize (Fig S2), giving additional credence to the co-localization and distribution pattern (cell poles and periphery) of PipX-GFP/PII-YFP foci.

If the dynamic localization of PipX-GFP and PII-YFP proteins during LD cycles was subjected to circadian control, foci would appear in LD entrained cells under the subjective night (that is, maintaining light when cultures should be switched to the next period of darkness), and additionally, foci formation and/or regulation would also be affected by interference with components of the circadian clock. To gain insights into this possible connection, S. elongatus cells expressing the fusion proteins were entrained with two LD (12:12) cycles and then released in 
constant light. Since foci did not reappear during the subjective night (Fig 2B), the results indicate that formation of the PipX-GFP/PII-YFP foci depends on darkness and not on circadian rhythms.

To further exclude regulatory connections with the circadian clock output pathway, the impact of cikA, sasA and rpaA null mutations in PipX-GFP/PII-YFP foci was investigated. Similar types and number of foci were detected in all three cases after 10 hours of darkness (Fig S4), thus excluding a role of the corresponding histidine kinases (CikA and SasA) or response regulator (RpaA) in the dynamic localization of PipX-GFP or PII-YFP. Therefore, the PipX-GFP/PII-YFP foci respond to environmental cues with no apparent connection with the circadian clock.

Next, we confirmed that visualization of foci did not require the simultaneous presence of the two fluorescent proteins. In S. elongatus cultures expressing either PipX-GFP or PII-YFP, $80 \%$ or $55 \%$ of the cells, respectively, showed at least one focus (compare PipX-GFP and PII-YFP panels in Fig 3), with localization patterns very similar to the previously obtained by co-expression of PII-YFP and PipX-GFP proteins. Since wild type PII or PipX and their corresponding fusion protein derivatives were co-expressed from independent genomic locations, their association would mainly depend on their relative affinity. In this context, the high affinity between PII subunits would favor intracellular accumulation of homotrimers (PII or PII-YFP), while the monomeric nature of PipX may result in a greater proportion of the PII-PipX complexes having one or more PipX-GFP subunits, that would be fluorescent in cells co-expressing PipX and PipX-GFP.

\section{PII, but not PipX, is essential for foci formation at dark}

To investigate the determinants of foci formation, we next introduced pipX null alleles into $S$. elongatus strains expressing PipX-GFP or PII-YFP. The inactivation of pipX had no significant impact on the distribution of PipX-GFP or PII-YFP foci within cells (Fig 3, micrographs from the second column, heatmaps and histograms), indicating that PipX plays no role in the process of foci 
formation other than binding to PII (or PII-YFP) proteins, which therefore anchor PipX to the foci. On the other hand, the proportion of cells with PipX-GFP foci increased slightly in the pipX mutant (compare histograms in Fig 3), a result probably reflecting competition between PipX-GFP and PipX in the context of formation of PII-PipX complexes, and arguing against PipX-GFP foci being artifacts caused by an excess of PipX proteins.

Next, we attempted introduction of null $g \ln B$ alleles into $S$. elongatus strains expressing PipX-GFP or PII-YFP. However, increasing the intracellular PipX/PII ratio is toxic to S. elongatus and therefore $g \ln B$ inactivation requires previous loss of function or down regulation of pipX (Espinosa et al., 2009; Espinosa et al., 2010; Chang et al., 2013). In line with this, we could not obtain a $g \ln B$ null mutant in WT strains expressing PipX-GFP, while $p i p X g \ln B$ PipX-GFP clones grew slowly, produced abnormally large cells (Fig S5) and lost viability when subcultured, forcing us to perform the confocal microscopy analyses (involving a 8 hours dark pulse) with freshly obtained cultures. Interestingly, neither of the three viable strains expressing PII-YFP or PipX-GFP in $g \ln B$ null backgrounds ( $g \ln B$ PII-YFP, $p i p X g \ln B$ PipX-GFP or pipXglnB PII-YFP) made foci in the dark (Fig 3, micrographs from the first column), indicating that wild type PII is required to induce foci formation, and that the YFP insertion at the C-terminus interferes with this ability.

\section{PII-YFP retains essential functions of PII: interactions with PipX}

The compromised phenotype of pipXglnB PipX-GFP indicates that PipX-GFP also conferred toxicity in the absence of PII. Although we cannot exclude that the levels of PipX-GFP expressed from pipXglnB PipX-GFP cells were slightly lower than the levels of PipX protein in WT $S$. elongatus, where $g \ln B$ can not be inactivated, we think more likely that the GFP tag decreased PipX toxicity, presumably by decreasing interactions with relevant protein target(s).

Interestingly, inactivation of $g \ln B$ was straightforward in cells expressing PII-YFP both in the WT and in the pipX mutant (Fig S1B S1D), indicating that the PII-YFP fusion protein is able to 
provide essential functions related to the ability to counteract the toxic effect of PipX, presumably by sequestering it into PII-PipX complexes.

Next, we compared the effect of $g \ln B$, $p i p X$ and $g \ln B-g f p$ alleles on the growth of $S$. elongatus on ammonium- or nitrate-containing media under both continuous light (LL) and LD cycles. As shown in Fig. 4, these analyses were in line with the known association of PII deficiency with poor growth in ammonium-containing media (Forchhammer and Tandeau de Marsac, 1995; Chang et al., 2013), as well as with our failure to associate PipX deficiency with growth defects (Espinosa et al., 2007; Chang et al., 2013). In addition, the results revealed phenotypic defects of strain $g \ln B$ PII-YFP on both nitrate and ammonium-containing media under LD cycles. Interestingly, the differences between $g \ln B$ PII-YFP and control strains on ammonium-containing media under LL conditions were abolished by growing them at lower light intensities. Since previous phenotypic studies of $g \ln B$ mutants only focused on the $\mathrm{C} / \mathrm{N}$ balance sensing function of PII, the results resported here are indicative of a greater regulatory complexity.

The finding that all $g \ln B$ PII-YFP growth phenotypes detected here were completely suppressed by inactivation of pipX (compare $\mathrm{B}^{-} \mathrm{PII-YFP}$ and $\mathrm{X}^{-} \mathrm{B}^{-}$PII-YFP in Fig.4) emphasizes the in vivo importance of PII to counteract PipX activity under different environmental conditions. In this context, it is worth noting that PII (or PII-YFP) also counteract PipX toxicity under conditions in which NtcA is known to be mainly inactive, as it is the case in ammonium cultures, thus supporting our previous claims of PipX having NtcA-independent functions (Espinosa et al., 2014).

\section{PipX-GFP mediates regulated partner swapping between PII and NtcA in S. elongatus}

To demonstrate that the localization of PipX-GFP into foci was not an artefact, but relied on the regulated formation of PII-PipX complexes, we next engineered and subsequently analysed in WT and pipX null backgrounds PipX-GFP and its derivatives PipX ${ }^{\mathrm{E} 4 \mathrm{~A}}-\mathrm{GFP}$ and PipX ${ }^{\mathrm{Y} 32 \mathrm{~A}}-\mathrm{GFP}$. The later proteins carry amino acid substitutions that impair interactions between PipX and PII, with 
Y32A having more drastic effects on PII binding than E4A (Espinosa et al., 2009; Laichoubi et al., 2012; Espinosa et al., 2014; Labella et al., 2016). In complete agreement with the specific effects of the corresponding point mutations on the binding to PII, cells expressing PipX ${ }^{\mathrm{E} 4 \mathrm{~A}}-\mathrm{GFP}$ or, to a much greater extent, PipX ${ }^{\mathrm{Y} 32 \mathrm{~A}}$-GFP, reduced the number of cells with foci (Fig 5A). In addition, the comparatively fewer PipX ${ }^{\mathrm{E} 4 \mathrm{~A}}$-GFP foci observed in WT than in pipX null backgrounds agreed with the expected competition for PII binding between endogenous PipX and a mutant derivative retaining significant affinity for PII.

To obtain additional evidence of the functionality of PipX-GFP in the context of its role as NtcA coactivator in S. elongatus, we determined the ability of PipX-GFP to stimulate bleaching under nitrogen starvation. As shown in Fig 5B, expression of PipX-GFP restored the WT phenotype to the otherwise non-bleaching pipX mutant (Espinosa et al., 2007), thus indicating that PipX-GFP retains the ability to interact with $\mathrm{NtcA}$ in a regulated manner, that is, is able to perform partner swapping in vivo between PII and NtcA in response to the appropriate metabolic signals. Therefore, all available evidence is consistent with PipX-GFP being able to substitute for PipX in the context of the nitrogen regulatory network.

\section{PII-PipX foci depend on a low ATP/ADP ratio. Biochemical and genetic evidences}

In the obligate phototroph S. elongatus, energy is primarily obtained by cells growing in the light, and changes in culture illumination affect the relative abundance of adenine nucleotides (Rust et al., 2011). To investigate a cause-effect relationship between the energy status and the darkdependent formation of PII-PipX foci, we subjected S. elongatus cultures to LD (12:12) during a period of 28 hours. The ratio of ATP to ADP + ATP (hereafter ATP/ADP ratio) decreased progressively during the dark period, to ca $25 \%$ of the initial level, recovering very rapidly after illumination was restored (Fig 6A). Importantly, the changes of the ATP/ADP ratio always preceded, and were inversely correlated with, changes in the number of foci during LD cycles (Figs 6A and 
2B). To further substantiate the cause-effect relationship, we next perturbed the ATP/ADP ratio by biochemical or genetic manipulation of S. elongatus cells and determined the impact of the corresponding changes on the presence of foci.

To decrease the ATP/ADP ratio under conditions in which we do not normally observe foci, that is, in illuminated cultures, we used DCCD, a specific inhibitor of the $\mathrm{F}_{0} \mathrm{~F}_{1}$-ATP synthase (Fig 6B) (Takano et al., 2015). Foci were first detected 30 min after DCCD treatment, following a fast and sharp decrease in the ATP/ADP ratio, and continued to increase during the following 4 hours of the experiment, when ca. $60 \%$ of the analysed cells had detectable foci (Figs 6C and D).

The Escherichia coli sugar symporter GalP confers to S. elongatus the ability to transport and metabolize glucose in the dark (McEwen et al., 2013). To increase the ATP/ADP ratio in darkness, we introduced the transcriptional fusion $\mathrm{P}_{\text {trc }}:$ galP and the lacI repressor gene into $S$. elongatus, following a previously described approach (Pattanayak et al., 2015). This resulted in a strain (PtrcGalP) capable of heterotrophic growth determined by the IPTG-dependent uptake of glucose, as confirmed by the $\sim 40 \%$ increase in biomass of Ptrc-GalP cells fed with glucose relative to the control strains (Fig 6E and F). In parallel, the ATP/ADP ratios in Ptrc and Ptrc-GalP cultures, each with or without glucose, were determined after 8 hours in the dark (Fig 6G). A high ATP/ADP ratio was only observed for Ptrc-GalP cells fed with glucose ( $\sim 80 \%$, versus $\sim 40-50 \%$ for controls). In this genetic background the addition of glucose significantly reduced the number of cells with PipX-GFP foci (Figs $6 \mathrm{H}$ and I). In particular, PipX-GFP foci were detected in $\sim 12 \%$ and $\sim 80 \%$, respectively, of the cells from cultures with or without glucose.

Taken together, the results show that the ATP/ADP ratio inversely correlates with the amount of foci, indicating that the spatio-temporal localization of PII and PipX into foci is regulated by the energy charge, with foci formation at dark being in complete agreement with the stimulatory role of ADP on PII-PipX complex formation in vitro (Zeth et al., 2014) 


\section{Environmental importance of PII-PipX complexes and possible significance of their localization into foci}

In S. elongatus, the levels of both PII and PipX proteins remain constant along LD cycles (Guerreiro et al., 2014), but PII is far more abundant than PipX (Fig. 1), suggesting that at the relatively high ADP levels detected at darkness most of the PipX protein could be trapped into PIIPipX complexes. The energy-dependent co-localization of PipX and PII into foci demonstrated here (Fig. 6) appears to be a visual manifestation of the abundance of PII-PipX complexes and of their preference for peripheral and polar localization.

Sequestering of PipX by PII at low ATP/ADP ratios would interfere with energetically expensive biological processes, decreasing transcriptional activity in the dark (Hosokawa et al., 2011; Takano et al., 2015). In addition to down regulation of NtcA target genes co-regulated by PipX (Espinosa et al., 2007; Valladares et al., 2011; Espinosa et al., 2014; Giner-Lamia et al., 2017), it would also affect the predicted signalling pathway responsible for the toxic functions of PipX in the absence of PII (Fig. 4 and (Espinosa et al., 2009; Espinosa et al., 2010; Chang et al., 2013)). Thus, PII sequestering of PipX may be particularly important under environmental conditions in which the cells face energy shortage. In addition, it would ensure the immediate liberation of PipX in response to rapid changes in the levels of ATP/ADP induced by the transition from dark to light (Fig. 6A). In this context, a recent work in S. elongatus called attention to the importance of light-dependent or light-induced activities specifically during the lag phase occurring after the transition from dark to light (Watanabe et al., 2015), and it is tempting to speculate on the implication of PipX, a signalling protein exclusive of cyanobacteria, in such processes.

The localization of PII-PipX complexes into peripherally located foci appears reminiscent of the recently described membrane microdomains that are involved in organizing signalling networks in B. subtilis (Schneider et al., 2015). In the light of the results obtained here, we propose that PIIPipX complexes are involved in a signalling pathway that transmits signals of energy abundance to 
essential cell processes, facilitating adaptation to the new environmental conditions. PII would be responsible for the spatial localization of PipX as well as preventing PipX interaction with its additional target(s) at low ATP/ADP ratios.

In summary, the interplay of regulatory interactions in which PII and PipX participate would play circadian-independent roles in the attenuation of transcriptional activity and other functions at darkness, while facilitating the return to the essential, and energy costly, light-dependent or lightinduced activities.

\section{EXPERIMENTAL PROCEDURES}

\section{Molecular genetic techniques and growth conditions}

Cloning procedures were carried out with Escherichia coli DH5a, using standard techniques (Sambrook et al., 1989). All the constructs were analysed by automated dideoxy DNA sequencing. Strains and plasmids are described, together with the used oligonucleotides, in Tables S1 and S2.

S. elongatus strains were routinely grown photoautotrophically at $30^{\circ} \mathrm{C}$ while shaking under constant illumination $\left(40 \mu \mathrm{E}\left(\mu \mathrm{mol}\right.\right.$ photons $\left.\left.\mathrm{m}^{-2} \mathrm{~s}^{-1}\right)\right)$ provided by cool white fluorescent lights. Media used were blue-green algae media BG11 (BG11 $1_{0}$ plus $17.5 \mathrm{mM} \mathrm{NaNO}$ and $10 \mathrm{mM}$ HEPES/NaOH pH 7.8) and $\mathrm{BG}_{1} 1_{\mathrm{A}}\left(\mathrm{BG} 11_{0}\right.$ plus $5 \mathrm{mM} \mathrm{NH}_{4} \mathrm{Cl}$ and $\left.5 \mathrm{mM} \mathrm{HEPES} / \mathrm{NaOH} \mathrm{pH} 7.8\right)$ and BG11 $1_{0}$. For growth on plates the media was solidified by addition of $1.5 \%(\mathrm{w} / \mathrm{v})$ agar. Plates were routinely incubated at $30^{\circ} \mathrm{C}$ under constant illumination. S. elongatus strains were transformed essentially as described by (Golden and Sherman, 1984). Whenever used, antibiotic concentrations (in $\mu \mathrm{g} \mathrm{m}{ }^{-1}$ ) were: kanamycin/5, streptomycin/2, nourseothricin/2.5 and chloramphenicol/5. $\mathrm{F}_{\mathrm{o}} \mathrm{F}_{1^{-}}$ATP synthase inhibitor DCCD (N,N-dicyclohexylcarbodiimide), dissolved in DMSO, was supplemented when indicated at $10 \mu \mathrm{M}$.

Liquid cultures of strains were pre-grown in continuous light in a medium lacking antibiotics. When required, cell optical density was recorded at $750 \mathrm{~nm}\left(\mathrm{OD}_{750}\right)$. Cultures were diluted to $\mathrm{OD}_{750}$ $\sim 0.2$ and grown either in constant light or in 12 hours light $(29 \mu \mathrm{E}) / 12$ hours dark (LD) cycling 
conditions at $30^{\circ} \mathrm{C}$. When necessary, cells were entrained to two 12:12 LD cycles prior to be released in constant light. For the experiments performed with Ptrc-GalP strains, cells were grown in BG-11 medium with $100 \mu \mathrm{M}$ IPTG with or without supplementation with $0.5 \%(\mathrm{w} / \mathrm{v})$ glucose.

To test growth on solid media, exponentially growing cultures were adjusted to $0.5\left(\mathrm{OD}_{750}\right)$ before dropping $5 \mu \mathrm{L}$ of the cell suspensions and two different dilutions (1/5 and 1/10) onto BG11 or BG11 $1_{\mathrm{A}}$ plates. Plates were incubated in LL at two light regimens: 27 or $1.2 \mu \mathrm{E}$ for 3 or 12 days, respectively. Plates in LD 12:12 (15 $\mu \mathrm{E}$ in the light period) were incubated 6 days. Plates were photographed after incubation times.

\section{Microscopy, image acquisition and analysis}

Cells were photographed with a Leica confocal microscope (running under Leica Confocal Software version 2.61, Leica Microsystems) as described (Labella et al., 2017). Exponentially growing cells $(5 \mu \mathrm{L})$ were mounted on $1 \%$ low-melting point agarose pads for microscopy. Filter specificities were as follow: ex633, TD 488/543/633, em665-700 (for cyanobacterial auto fluorescence analysis); ex488, TD 488/543/633, em495-537 (for GFP and ZsGreen analyses) and ex514, DD 458/514, em540-580 (for EYFP analysis). Whenever indicated settings were modified to minimize the bleed-through of GFP signal into EYFP emission channel and vice-versa were as follow: ex633/em665-700 (for cyanobacterial auto fluorescence analysis); ex476/em486-520 (for ZsGreen/GFP) and ex514/em540-580 (for EYFP). When required, cells were fixed with $1 \%$ formaldehyde for $20 \mathrm{~min}$ at RT, centrifuged and washed twice in PBS buffer and finally store at $4^{\circ} \mathrm{C}$ until needed.

To determine foci position both, cells and foci, were automatically detected using a homemade

python script (available on https://dfgm.ua.es/genetica/investigacion/cyanobacterial_genetics/Resources.ht ml). Foci coordinates, referenced to their cell center and normalized by their cell length and width, were used to generate a density distribution heatmap corresponding to a cell quarter. When 
represented in figures, heatmaps correspond to 4 identical cell quarter maps. Note that in cells coexpressing PipX-GFP and PII-YFP, heatmaps correspond to signals from PipX-GFP foci.

\section{Quantification of intracellular ATP and ADP}

Quantification of intracellular ATP and ADP was carried out as described (Rust et al., 2011), ATP and ADP extractions from intact cyanobacterial cells were as described (Sunamura et al., 2010). For ATP content determination, thawed extracts $(100 \mu \mathrm{L})$ were diluted $\times 1 / 2.6$ in $\mathrm{L}$ buffer $(25 \mathrm{mM}$ $\mathrm{KCl}, 50 \mathrm{mM} \mathrm{MgSO} 4,1 \mathrm{mM}$ PEP and $100 \mathrm{mM}$ HEPES, $\mathrm{pH}$ 7.4) and samples were loaded into the wells of a black plastic 96-well plate (Nunc; Thermo Scientific, Waltham, USA). As internal standards, 0, 20, 40, 60, 80 and $100 \mathrm{nM}$ of ATP was preloaded on additional wells. Luminescence reaction was initiated by addition of $40 \mu \mathrm{L}$ of a luciferase mixture $\left(12 \mu \mathrm{g} \mathrm{m}{ }^{-1}\right.$ luciferase (firefly recombinant), $250 \mu \mathrm{M}$ D-luciferin, and $1 \mathrm{mM}$ DTT in Reaction buffer) (Invitrogen; Thermo Scientific, USA) to each sample. Plates were shaken in the dark for 30 seconds at $28{ }^{\circ} \mathrm{C}$ before measuring the luminescence signal from each well with a microplate reader (Perkin Elmer Victor3). The ATP content of each sample was determined by linear regression from the internally standardized wells.

To measure ADP + ATP, extracts were diluted as above, except that $3 \mathrm{U} \mathrm{ml}^{-1}$ of type II pyruvate kinase from rabbit muscle (Roche) added to catalyse the conversion of ADP to ATP. As internal standards, 0, 20, 40, 60, 80 and $100 \mathrm{nM}$ of ADP were used. Extracts and standards were incubated for 30 minutes at $37^{\circ} \mathrm{C}$ and 10 minutes at $90^{\circ} \mathrm{C}$ to inactivate enzymes. ATP content was determined as described above and the ADP content of the original sample was calculated as the difference between the reaction with pyruvate kinase (“ATP + ADP”) and the reaction without pyruvate kinase (“ATP”), using the linear regression from the ADP converted to ATP standards. 


\section{Determination of phycocyanin content}

A whole-cell absorbance spectrum was carried out to estimate pigment contents as described (Espinosa et al., 2007). Pigment content was calculated based on absorbance maxima at $631 \mathrm{~nm}$ for phycocyanin. Pictures were taken after 48 hours of initiation of nitrogen deprivation.

\section{Construction of plasmids and strains}

To express the PipX-GFP fusion protein from the pipX promoter, plasmid pUAGC817 carrying the pipX-gfp translational fusion flanked by S. elongatus Neutral Site 1 (NS1) (Bustos and Golden, 1992), was engineered. First, pipX sequences comprising the complete coding region plus $121 \mathrm{bp}$ from its own promoter were amplified from genomic DNA using primers PipX-gfp-1F/PipXgfp-2R, cut with EcoRI and cloned into plasmid pUAGC816 (carrying GFPmut2), yielding plasmid pUAGC817. To construct PipX-GFP variants carrying point mutations E4A or Y32A, pipX point mutant sequences were amplified from pUAGC375 and pUAGC380 with primers Up-pipX-ApaI1F/pipX-XmaJI-1R, the product cut with ApaI/XmaJI and cloned into pUAGC816, yielding plasmids pUAGC895 (PipX $\left.{ }^{\mathrm{E} 4 \mathrm{~A}}-\mathrm{GFP}\right)$ and pUAGC896 (PipX $\left.{ }^{\mathrm{Y} 32 \mathrm{~A}}-\mathrm{GFP}\right)$.

To express PII-YFP fusion protein either individually or together with PipX-GFP, first $y f p$ coding sequences were amplified from pEYFP-C1 with primers EFP-1F/EFP-1R, cut with SpeI/NotI and cloned into pUAGC816 and pUAGC817, yielding plasmids pUAGC827 and pUAGC828, respectively. Next, glnB sequences comprising the coding region plus $187 \mathrm{bp}$ from its own promoter were amplified using primers glnB-EFP-1F/glnB-EFP-1R, cut with SpeI/MluI and cloned into pUAGC828 and pUAGC827 yielding, respectively, plasmids pUAGC830 and pUAGC838.

To express ZsG-KaiC fusion protein, first the ZsGreen coding gene was amplified from pZsGreen1-N1 with primers Green1-F/Green1-R and the product cut with EcoRI/SmaI and cloned into pUAGC280, yielding plasmid pUAGC893. kaiC coding sequences were amplified from 
genomic DNA with primers KaiC-F/KaiC-R cut with BamHI/XhoI and cloned into pUAGC893, yielding plasmid pUAGC894.

To express the galP gene from Neutral Site 3 (NS3) in S. elongatus, first nat gene sequence was PCR-amplified with primers nat1ALR-1F/nat1ALR-1R and cloned into blunt-end pBluescriptSk+, yielding plasmid pBlue-Nt. NS3 regions were amplified according to (Niederholtmeyer et al., 2010) using primer pairs NS3-Left-1F/NS3-Left-1R and NS3-Right1F/NS3-Right-1R. The first NS3 amplicon was cut with KpnI/ApaI and cloned into pBlue-Nt, yielding intermediary plasmid pBlue-Nt-NS3L. The second NS3 amplicon was digested with Xbal/SacI and cloned into plasmid pBlue-Nt-NS3L, yielding plasmid pUAGC49. Next, a 1581 bp fragment containing lacI and Ptrc sequences was excised from pUAGC280 with EcoRI/XmnI and cloned into pUAGC49, producing plasmid pUAGC70. Finally, galP coding sequences were amplified from E. coli genomic DNA using GalP-rev-XbaI/Ptrc-galP-1F, cut with NcoI/XbaI and cloned downstream the Ptrc promoter of pUAGC70, producing pUAGC74.

To express PII-YFP fusion protein from NS3, $g \ln B-y f p$ coding sequences (including its own promoter) were cut with EcoRI/XmnI from plasmid pUAGC838 and cloned into EcoRI/Ecl136II digested pUAGC70 resulting in the replacement of lacI $\mathrm{P}_{t r c}$ sequences from pUAGC70 by $g \ln B-y f p$ sequences and yielding plasmid pUAGC901.

For expression in S. elongatus of PipX-GFP and/or PII-YFP, plasmids pUAGC817 and pUAGC838 were independently transformed into wild type, pipX and pipXglnB strains, and pUAGC838 was transformed into wild type, sasA, cikA and rpaA strains. Clones were selected on plates supplemented with streptomycin and the correct insertion into the NS1 was confirmed by PCR with 7942NSIA-F/NSI-1R. For expression of PipX-GFP point mutants $\left(\mathrm{PipX}^{\mathrm{E} 4 \mathrm{~A}}-\mathrm{GFP}\right.$ or PipX ${ }^{\mathrm{Y} 32 \mathrm{~A}}$ GFP) plasmids pUAGC895 and pUAGC896, were transformed into WT or pipX strains and transformants selected and analyzed as described above. 
For expression of ZsG-KaiC, plasmid pUAGC894 was transformed into S. elongatus, clones selected on streptomycin containing plates and the correct integration into NS1 verified as described above. For co-expression of ZsG-KaiC and PII-YFP, ZsG-KaiC strain was transformed with pUAGC901 and selection was carried out on nourseothricin plates. Correct integration of the construct into NS3 was verified by PCR with NS3-seq-1F/NS3-seq-1R. For expression of GalP, pUAGC74 was transformed into WT, PipX-GFP, and PipX-GFP PII-YFP strains. Transformants were selected on nourseothricin plates and clones analysed for correct integration of the construct into NS3 as described above.

Inactivation of the $g \ln B$ gene in both PII-YFP and pipX strains was carried out by transformation with plasmid pPM128 and selection of kanamycin resistant clones. Complete segregation of $g \ln B$ alleles was confirmed by PCR analysis with primers glnB-EFP-1F/GlnBOV-7942-1R. To exclude inactivation of the $g \ln B$ sequences fused to $y f p$, an additional PCR was performed with NSI-1F/ glnB-EFP-1R.

\section{Immunodetection methods}

$15 \mathrm{ml}$ samples of $S$. elongatus cells were harvested by centrifugation at $7300 \mathrm{~g}$ for $5 \mathrm{~min}$. The pellet was resuspended in $100 \mathrm{ml}$ lysis buffer $(50 \mathrm{mM}$ Tris/HCl, $\mathrm{pH}$ 7.4, $4 \mathrm{mM}$ EDTA, $0.5 \mathrm{mM}$ PMSF, 0.5 $\mathrm{mM}$ benzamidine, $1 \mathrm{mM}$ DTT) and $100 \mathrm{~nm}$ glass beads were added. The mixture was homogenized with two cycles of $60 \mathrm{~s}$ in a Minibeadbeater. After centrifugation (5500 g for $5 \mathrm{~min})$, the supernatant fraction (crude protein extract) was transferred to a new tube. Protein concentrations were estimated by Lowry (Bio-Rad RC DC reagents). Protein immunodetection was performed on protein-loaded PVDF membrane, transferred from acrylamide gels using a wet system. Membrane was blocked with TBS (20 mM Tris, $\mathrm{pH} 7.5,500 \mathrm{mM} \mathrm{NaCl}$ ) solution containing $5 \% \mathrm{BSA}$ for $1 \mathrm{~h}$ at room temperature, and incubated overnight in TBS solution containing $2 \%$ BSA and the primary antibody (a 1:10.000 dilution for anti-PII, 1:10.000 for anti-GFP and 1:7500 for anti-PipX). The membrane was then incubated with ECL rabbit IgG, HRP-linked $\mathrm{F}\left(\mathrm{ab}^{\prime}\right)_{2}$ fragment (from donkey) (GE Healthcare) 
(1:150000 dilution). Immunoreactive bands were detected using the Supersignal West Femto (Thermo) and X-ray films. Antisera against PII and PipX were produced in rabbits (Pineda Antikorper Service, Berlin, Germany) and kindly provided by Karl Forchhammer. An anti-GFP, Nterminal antibody produced in rabbit, was purchased from (Sigma-Aldrich).

\section{ACKNOWLEDGEMENTS}

We thank A. Llop and C.V. Racovac for technical and experimental support, A. Obrebska for plasmids, K. Forchhammer for PII and PipX antisera and R. Dixon for constructive discussions. The authors thank the Spanish and Valencian Governments for supporting grants (Gerónimo Forteza contract FPA/2015/052 to J.I.L) and (BFU2012-33364 and BFU2015-66360-P to A.C), and the Research technical Services of the University of Alicante (SSTTI-UA).

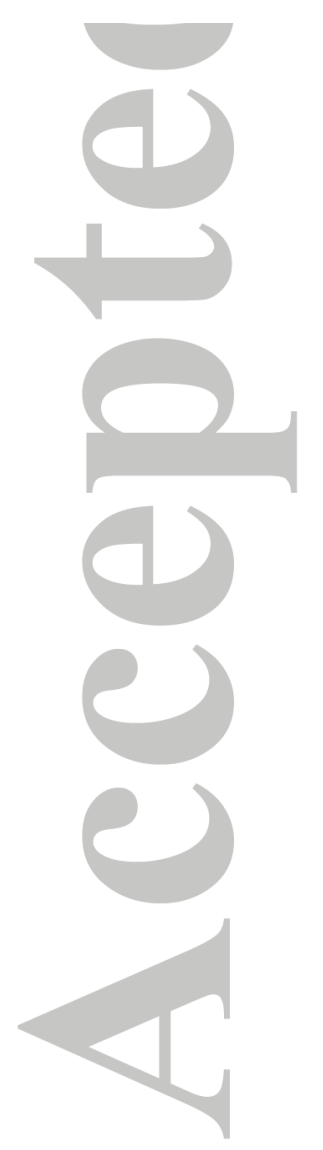




\section{REFERENCES}

Boyd, J.S., Cheng, R.R., Paddock, M.L., Sancar, C., Morcos, F., and Golden, S.S. (2016) A Combined Computational and Genetic Approach Uncovers Network Interactions of the Cyanobacterial Circadian Clock. J Bacteriol 198: 2439-2447.

Burillo, S., Luque, I., Fuentes, I., and Contreras, A. (2004) Interactions between the nitrogen signal transduction protein PII and N-acetyl glutamate kinase in organisms that perform oxygenic photosynthesis. J Bacteriol 186: 3346-3354.

Bustos, S.A., and Golden, S.S. (1992) Light-regulated expression of the psbD gene family in Synechococcus sp. strain PCC 7942: evidence for the role of duplicated psbD genes in cyanobacteria. Mol Gen Genet 232: 221-230.

Cohen, S.E., Erb, M.L., Selimkhanov, J., Dong, G., Hasty, J., Pogliano, J., and Golden, S.S. (2014) Dynamic localization of the cyanobacterial circadian clock proteins. Curr Biol 24: 1836-1844.

Chang, Y., Takatani, N., Aichi, M., Maeda, S., and Omata, T. (2013) Evaluation of the effects of $P_{I I}$ deficiency and the toxicity of PipX on growth characteristics of the $\mathrm{P}_{\mathrm{II}}$-less mutant of the cyanobacterium Synechococcus elongatus. Plant Cell Physiol 54: 1504-1514.

Chellamuthu, V.R., Alva, V., and Forchhammer, K. (2013) From cyanobacteria to plants: conservation of PII functions during plastid evolution. Planta 237: 451-462.

Espinosa, J., Forchhammer, K., and Contreras, A. (2007) Role of the Synechococcus PCC 7942 nitrogen regulator protein PipX in NtcA-controlled processes. Microbiology 153: 711-718.

Espinosa, J., Forchhammer, K., Burillo, S., and Contreras, A. (2006) Interaction network in cyanobacterial nitrogen regulation: PipX, a protein that interacts in a 2-oxoglutarate dependent manner with PII and NtcA. Mol Microbiol 61: 457-469.

Espinosa, J., Castells, M.A., Laichoubi, K.B., and Contreras, A. (2009) Mutations at pipX suppress lethality of PII-deficient mutants of Synechococcus elongatus PCC 7942. J Bacteriol 191: 48634869.

Espinosa, J., Castells, M.A., Laichoubi, K.B., Forchhammer, K., and Contreras, A. (2010) Effects of spontaneous mutations in PipX functions and regulatory complexes on the cyanobacterium Synechococcus elongatus strain PCC 7942. Microbiology 156: 1517-1526.

Espinosa, J., Boyd, J.S., Cantos, R., Salinas, P., Golden, S.S., and Contreras, A. (2015) Cross-talk and regulatory interactions between the essential response regulator $\mathrm{RpaB}$ and cyanobacterial circadian clock output. Proc Natl Acad Sci U S A 112: 2198-2203.

Espinosa, J., Rodriguez-Mateos, F., Salinas, P., Lanza, V.F., Dixon, R., de la Cruz, F., and Contreras, A. (2014) PipX, the coactivator of NtcA, is a global regulator in cyanobacteria. Proc Natl Acad Sci U $S$ A 111: E2423-E2430.

Feria Bourrellier, A.B., Valot, B., Guillot, A., Ambard-Bretteville, F., Vidal, J., and Hodges, M. (2010) Chloroplast acetyl-CoA carboxylase activity is 2-oxoglutarate-regulated by interaction of PII with the biotin carboxyl carrier subunit. Proc Natl Acad Sci U S A 107: 502-507.

Flores, E., and A. Herrero (1994) The molecular biology of cyanobacteria. In. Bryant, D.A. (ed). Dordrecht ; Boston: Kluwer Academic Publishers, pp. 488-517.

Fokina, O., Chellamuthu, V.R., Forchhammer, K., and Zeth, K. (2010) Mechanism of 2-oxoglutarate signaling by the Synechococcus elongatus PII signal transduction protein. Proc Natl Acad Sci U S A 107: 19760-19765.

Forchhammer, K., and Tandeau de Marsac, N. (1995) Functional analysis of the phosphoprotein PII ( $g \ln B$ gene product) in the cyanobacterium Synechococcus sp. strain PCC 7942. J Bacteriol 177: 2033-2040.

Forchhammer, K., and Luddecke, J. (2016) Sensory properties of the PII signalling protein family. FEBS J 283: 425-437.

Gerhardt, E.C., Rodrigues, T.E., Muller-Santos, M., Pedrosa, F.O., Souza, E.M., Forchhammer, K., and Huergo, L.F. (2015) The bacterial signal transduction protein GlnB regulates the committed step 
in fatty acid biosynthesis by acting as a dissociable regulatory subunit of acetyl-CoA carboxylase. Molecular microbiology 95: 1025-1035.

Giner-Lamia, J., Robles-Rengel, R., Hernandez-Prieto, M.A., Muro-Pastor, M.I., Florencio, F.J., and Futschik, M.E. (2017) Identification of the direct regulon of NtcA during early acclimation to nitrogen starvation in the cyanobacterium Synechocystis sp. PCC 6803. Nucleic Acids Res 45: 1180011820 .

Golden, S.S., and Sherman, L.A. (1984) Optimal conditions for genetic transformation of the cyanobacterium Anacystis nidulans R2. J Bacteriol 158: 36-42.

Guerreiro, A.C., Penning, R., Raaijmakers, L.M., Axman, I.M., Heck, A.J., and Altelaar, A.F. (2016) Monitoring light/dark association dynamics of multi-protein complexes in cyanobacteria using size exclusion chromatography-based proteomics. Journal of proteomics 142: 33-44.

Guerreiro, A.C., Benevento, M., Lehmann, R., van Breukelen, B., Post, H., Giansanti, P. et al. (2014) Daily rhythms in the cyanobacterium Synechococcus elongatus probed by high-resolution mass spectrometry-based proteomics reveals a small defined set of cyclic proteins. Mol Cell Proteomics 13: $2042-2055$.

Hauf, W., Schmid, K., Gerhardt, E.C., Huergo, L.F., and Forchhammer, K. (2016) Interaction of the Nitrogen Regulatory Protein GlnB (PII) with Biotin Carboxyl Carrier Protein (BCCP) Controls Acetyl-CoA Levels in the Cyanobacterium Synechocystis sp. PCC 6803. Front Microbiol 7: 1700.

Heinrich, A., Maheswaran, M., Ruppert, U., and Forchhammer, K. (2004) The Synechococcus elongatus PII signal transduction protein controls arginine synthesis by complex formation with Nacetyl-L-glutamate kinase. Mol Microbiol 52: 1303-1314.

Hosokawa, N., Hatakeyama, T.S., Kojima, T., Kikuchi, Y., Ito, H., and Iwasaki, H. (2011) Circadian transcriptional regulation by the posttranslational oscillator without de novo clock gene expression in Synechococcus. Proc Natl Acad Sci U S A 108: 15396-15401.

Huergo, L.F., Chandra, G., and Merrick, M. (2013) PII signal transduction proteins: nitrogen regulation and beyond. FEMS microbiology reviews 37: 251-283.

Kobayashi, M., Rodriguez, R., Lara, C., and Omata, T. (1997) Involvement of the C-terminal domain of an ATP-binding subunit in the regulation of the ABC-type nitrate/nitrite transporter of the Cyanobacterium synechococcus sp. strain PCC 7942. J Biol Chem 272: 27197-27201.

Labella, J.I., Cantos, R., Espinosa, J., Forcada-Nadal, A., Rubio, V., and Contreras, A. (2017) PipY, a member of the conserved COG0325 family of PLP-binding proteins, expands the cyanobacterial nitrogen regulatory network. Frontiers in microbiology 8: 1244.

Labella, J.I., Obrebska, A., Espinosa, J., Salinas, P., Forcada-Nadal, A., Tremino, L. et al. (2016) Expanding the Cyanobacterial Nitrogen Regulatory Network: The GntR-Like Regulator PlmA Interacts with the PII-PipX Complex. Front Microbiol 7: 1677.

Laichoubi, K.B., Espinosa, J., Castells, M.A., and Contreras, A. (2012) Mutational analysis of the cyanobacterial nitrogen regulator PipX. PloS One 7: e35845.

Laichoubi, K.B., Beez, S., Espinosa, J., Forchhammer, K., and Contreras, A. (2011) The nitrogen interaction network in Synechococcus WH5701, a cyanobacterium with two PipX and two P(II)-like proteins. Microbiology 157: 1220-1228.

Lee, H.M., Flores, E., Herrero, A., Houmard, J., and Tandeau de Marsac, N. (1998) A role for the signal transduction protein PII in the control of nitrate/nitrite uptake in a cyanobacterium. FEBS Lett 427: 291-295.

Liu, Y., Tsinoremas, N.F., Johnson, C.H., Lebedeva, N.V., Golden, S.S., Ishiura, M., and Kondo, T. (1995) Circadian orchestration of gene expression in cyanobacteria. Genes Dev 9: 1469-1478.

Luddecke, J., and Forchhammer, K. (2015) Energy Sensing versus 2-Oxoglutarate Dependent ATPase Switch in the Control of Synechococcus PII Interaction with Its Targets NAGK and PipX. PloS one 10: e0137114. 
Llacer, J.L., Espinosa, J., Castells, M.A., Contreras, A., Forchhammer, K., and Rubio, V. (2010) Structural basis for the regulation of NtcA-dependent transcription by proteins PipX and PII. Proc Natl Acad Sci U S A 107: 15397-15402.

Llacer, J.L., Contreras, A., Forchhammer, K., Marco-Marin, C., Gil-Ortiz, F., Maldonado, R. et al. (2007) The crystal structure of the complex of PII and acetylglutamate kinase reveals how PII controls the storage of nitrogen as arginine. Proc Natl Acad Sci U S A 104: 17644-17649.

Markson, J.S., Piechura, J.R., Puszynska, A.M., and O'Shea, E.K. (2013) Circadian Control of Global Gene Expression by the Cyanobacterial Master Regulator RpaA. Cell 155: 1396-1408.

McEwen, J.T., Machado, I.M.P., Connor, M.R., and Atsumi, S. (2013) Engineering Synechococcus elongatus PCC 7942 for Continuous Growth under Diurnal Conditions. Applied and Environmental Microbiology 79: 1668-1675.

Merrick, M. (2014) Post-translational modification of P II signal transduction proteins. Front Microbiol 5: 763.

Niederholtmeyer, H., Wolfstadter, B.T., Savage, D.F., Silver, P.A., and Way, J.C. (2010) Engineering cyanobacteria to synthesize and export hydrophilic products. Appl Environ Microbiol 76: $3462-3466$.

Palanca, C., Pedro-Roig, L., Llacer, J.L., Camacho, M., Bonete, M.J., and Rubio, V. (2014) The structure of a PII signaling protein from a halophilic archaeon reveals novel traits and high-salt adaptations. FEBS J 281: 3299-3314.

Pattanayak, G.K., Lambert, G., Bernat, K., and Rust, M.J. (2015) Controlling the Cyanobacterial Clock by Synthetically Rewiring Metabolism. Cell Rep 13: 2362-2367.

Rust, M.J., Golden, S.S., and O'Shea, E.K. (2011) Light-driven changes in energy metabolism directly entrain the cyanobacterial circadian oscillator. Science 331: 220-223.

Sambrook, J., Fritsch, E., and Maniatis, T. (1989) Molecular cloning: a laboratory manual. Cold Spring Harbor, NY: Cold Spring Harbor Laboratory Press.

Schneider, J., Klein, T., Mielich-Suss, B., Koch, G., Franke, C., Kuipers, O.P. et al. (2015) Spatiotemporal remodeling of functional membrane microdomains organizes the signaling networks of a bacterium. PLoS Genet 11: e1005140.

Shultzaberger, R.K., Boyd, J.S., Diamond, S., Greenspan, R.J., and Golden, S.S. (2015) Giving Time Purpose: The Synechococcus elongatus Clock in a Broader Network Context. Annu Rev Genet 49: 485-505.

Sunamura, E., Konno, H., Imashimizu-Kobayashi, M., Sugano, Y., and Hisabori, T. (2010) Physiological impact of intrinsic ADP inhibition of cyanobacterial FoF1 conferred by the inherent sequence inserted into the gammasubunit. Plant Cell Physiol 51: 855-865.

Takano, S., Tomita, J., Sonoike, K., and Iwasaki, H. (2015) The initiation of nocturnal dormancy in Synechococcus as an active process. BMC Biol 13: 36.

Tanigawa, R., Shirokane, M., Maeda Si, S., Omata, T., Tanaka, K., and Takahashi, H. (2002) Transcriptional activation of NtcA-dependent promoters of Synechococcus sp. PCC 7942 by 2oxoglutarate in vitro. Proc Natl Acad Sci U S A 99: 4251-4255.

Valladares, A., Rodriguez, V., Camargo, S., Martinez-Noel, G.M., Herrero, A., and Luque, I. (2011) Specific role of the cyanobacterial PipX factor in the heterocysts of Anabaena sp. strain PCC 7120.J Bacteriol 193: 1172-1182.

Vazquez-Bermudez, M.F., Herrero, A., and Flores, E. (2002) 2-Oxoglutarate increases the binding affinity of the NtcA (nitrogen control) transcription factor for the Synechococcus glnA promoter. FEBS letters 512: 71-74.

Watanabe, S., Ohbayashi, R., Kanesaki, Y., Saito, N., Chibazakura, T., Soga, T., and Yoshikawa, H. (2015) Intensive DNA Replication and Metabolism during the Lag Phase in Cyanobacteria. PLoS One 10: e0136800.

Zeth, K., Fokina, O., and Forchhammer, K. (2014) Structural basis and target-specific modulation of ADP sensing by the Synechococcus elongatus PII signaling protein. J Biol Chem 289: 8960-8972. 
Zhao, M.X., Jiang, Y.L., Xu, B.Y., Chen, Y., Zhang, C.C., and Zhou, C.Z. (2010) Crystal structure of the cyanobacterial signal transduction protein PII in complex with PipX. J Mol Biol 402: 552-559.

\section{FIGURE LEGENDS}

Figure 1: Protein interactions at the cyanobacterial nitrogen regulatory network. (Left) PII and PipX interactions according to the concentration spectra of 2-OG and ATP/ADP ratios. Molecular players are illustrated as circles whose sizes, drawn to scale, refer to the amount of protein molecules according to (Guerreiro et al., 2014), taking into account their quaternary structure: 20078 PII trimers, 4560 PipX monomers and 1520 PipX trimers (dark and light coloured circle respectively), 510 NtcA dimers, 200 PlmA dimers, 160 NrtC monomers, 359 NAGK hexamers and 2409 BCCP monomers. The arrows go from hub to target proteins, with PipX being considered as a PII target. The position of the arrowheads (towards target proteins) indicates the conditions favouring the corresponding complexes, one of which is a ternary complex (PII-PipX-PlmA). Dashed lines indicate interactions identified by genetic means which have not been characterized at the molecular level.

Figure 2: PipX-GFP and PII-YFP localize into foci in darkness. (A) S. elongatus cells were imaged from cultures grown with nitrate in light or dark $(8 \mathrm{~h})$ conditions as indicated. The auto fluorescence (red), PipX-GFP (green), PII-YFP (yellow) and ZsG-KaiC (green) signals are shown as indicated. Where appropriated, heatmaps of PipX-GFP or ZsG-KaiC foci, with indication of the numbers detected, show their distribution relative to the cell auto fluorescence border. Colours represent $\%$ of normalized foci present at each cellular position. Histograms to the right show the $\%$ of cells with detectable GFP foci ( 0 to 4 ) and the number of cells per category. (B) (Left) Percentage of the cell population that showed PipX-GFP foci in diurnal conditions (light and dark conditions corresponding to yellow and gray regions, respectively). Cells were entrained to opposite light-dark cycles and sampled every 2 hours in either light (ZT 0-12) or dark (ZT 12-24). (Right) Percentage of the entrained cell population (after two cycles of $12 \mathrm{~h}$ light: $12 \mathrm{~h}$ dark) that showed foci after cells were released into continuous light (LL). The subjective night period is indicated with a grid. ZT (Zeitgeber time) refers to the time relative to "lights on".

Figure 3: Localization of PipX-GFP or PII-YFP in different genetic backgrounds. S. elongatus cells were imaged from cultures grown with nitrate in dark $(8 \mathrm{~h})$. Heatmaps and histograms 
correspond to the foci-forming strains (pictures from the second column). Examples of detected foci are indicated with arrowheads in the corresponding micrographs. Other details as in Fig. 2.

Figure 4: Effects of environmental and genetics backgrounds on S. elongatus survival to PipX toxicity. Exponentially growing cultures of $\mathrm{WT}$, pipX $\left(\mathrm{X}^{-}\right)$, pipXglnB $\left(\mathrm{X}^{-} \mathrm{B}^{-}\right), g \ln B$ PII-YFP $\left(\mathrm{B}^{-} \mathrm{PII}-\right.$

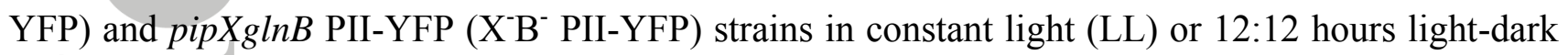
cycles (LD) were adjusted to $0,5\left(\mathrm{OD}_{750}\right)$ prior to drop $5 \mu 1$ of the cell suspension onto the plates (BG11 or BG11 $)$. Numbers on top and bottom refer to light intensities and culture dilutions, respectively. Pictures were taken after 6,12 and 3 days of incubation (from left to right) in the indicated light conditions.

Figure 5: In vivo interactions of PipX-GFP and point mutation derivatives indicate no interference of the GFP tag. (A) Comparative effect of PipX-GFP, PipX ${ }^{\mathrm{E} 4 \mathrm{~A}}-\mathrm{GFP}$ and $\mathrm{PipX}^{\mathrm{Y} 32 \mathrm{~A}}$ GFP on the number of cells with fluorescent foci in both WT and pipX strains. PipX structures (accession 2XG8), with the surface interacting with PII in blue and the position of mutated residues in red, illustrate the PipX-GFP derivative present in each strain. The distributions of cells with $(\geq 1$ focus, green bars) and without foci (black bars) for each strain in light (L) and dark (D) are shown. Total numbers of counted cells are indicated above each bar. (B) PipX-GFP rescues the nonbleaching phenotype of the pipX null mutant. (Top) Relative amount of phycocyanin content after nitrogen deprivation in WT, pipX and pipX PipX-GFP strains. (Bottom) Appearance of the different cultures after nitrogen deprivation (48 hours). The phycocyanin for pipX PipX-GFP corresponds to mean $\pm \mathrm{SD}$ of three independent clones ( $\mathrm{a}, \mathrm{b}$ and $\mathrm{c}$ ).

Figure 6: The amount of PipX-GFP /PII-YFP foci inversely correlates with the ATP/ADP ratio. (A) ATP/ADP ratio in S. elongatus cells subjected to the light and dark intervals indicated by white and gray regions, respectively. (B) Under light conditions the ATP/ADP ratio decreases upon addition of DCCD, an inhibitor of $\mathrm{F}_{1} \mathrm{~F}_{\mathrm{o}}$-ATPase. (C) Percentage of DCCD-treated cells with foci and relative $\%$ of ATP (referred to $\mathrm{t}_{0}$ set to $100 \%$ ) in light incubated cells (DMSO control) and after addition of DCCD. (D) Representative pictures of cells after 4 hours of treatment with or without DCCD (No foci were detected in the DMSO control). (E) Under dark conditions cells expressing GalP take up Glucose increasing their intracellular ATP/ADP ratio. (F) Growth curve from WT, Ptrc and Ptrc-GalP strains during light and dark conditions (yellow and gray intervals, respectively) with $100 \mu \mathrm{M}$ IPTG and with (filled symbols) or without (open symbols) glucose (Glc) supplementation. (G) ATP/ADP ratio in Ptrc and Ptrc-GalP strains after 10 hours in the dark in medium with or without glucose supplementation. (H) Percentage of cells with PipX-GFP foci in Ptrc-GalP strain 
after 10 hours of darkness with ( $n=1294$ cells) and without glucose ( $n=2169$ cells). (I) Representative micrographs of cells from $\mathrm{H}$.

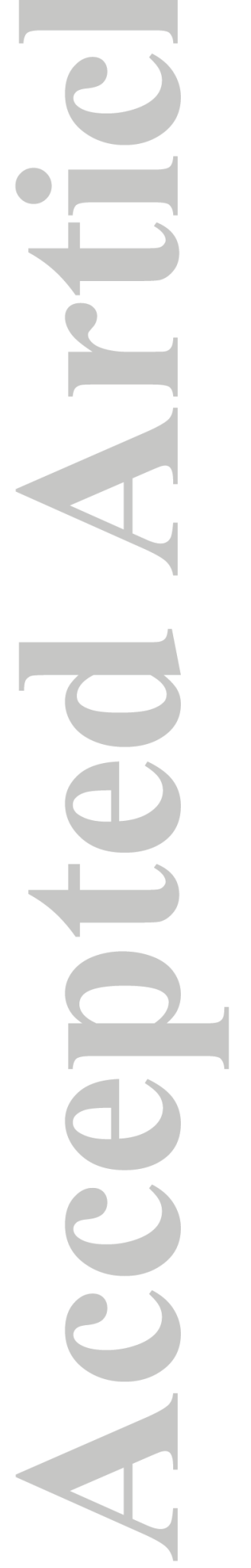




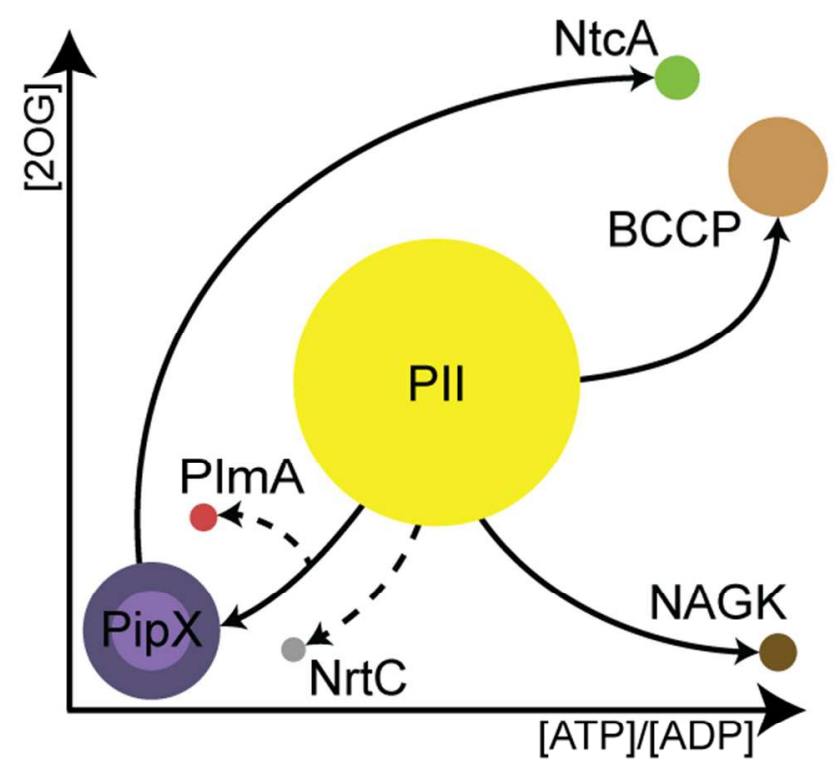

Figure 1: Protein interactions at the cyanobacterial nitrogen regulatory network. (Left) PII and PipX interactions according to the concentration spectra of 2-OG and ATP/ADP ratios. Molecular players are illustrated as circles whose sizes, drawn to scale, refer to the amount of protein molecules according to (Guerreiro et al., 2014), taking into account their quaternary structure: 20078 PII trimers, 4560 PipX monomers and 1520 PipX trimers (dark and light colored circle respectively), 510 NtcA dimers, 200 PImA dimers, 160 NrtC monomers, 359 NAGK hexamers and 2409 BCCP monomers. The arrows go from hub to target proteins, with PipX being considered as a PII target. The position of the arrowheads (towards target proteins) indicates the conditions favouring the corresponding complexes, one of which is a ternary complex

(PII-PipX-PImA). Dashed lines indicate interactions identified by genetic means which have not been characterized at the molecular level. 

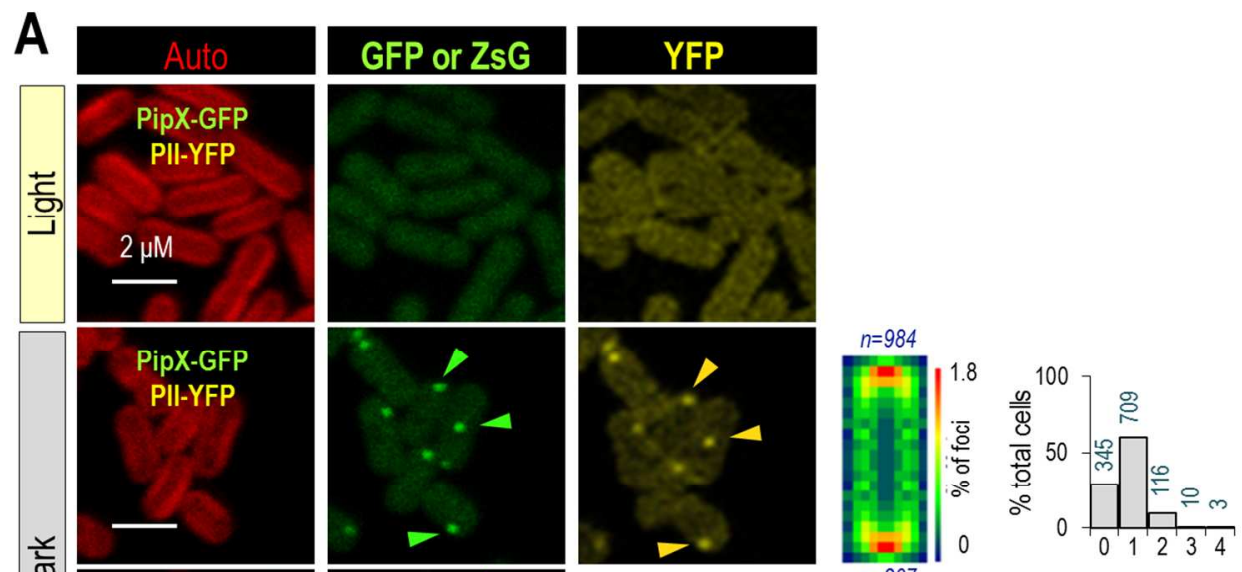

\section{ZsG-Kaic}
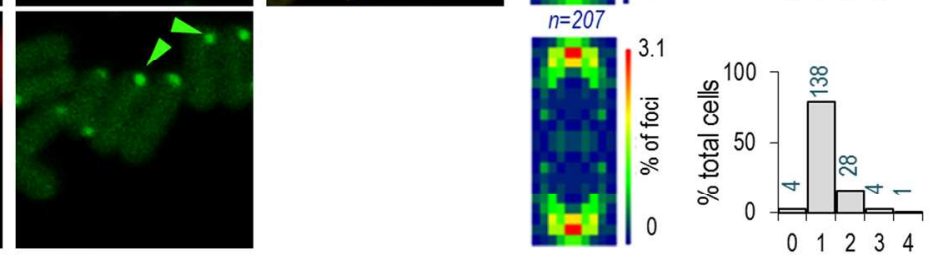

Number of foci/cell
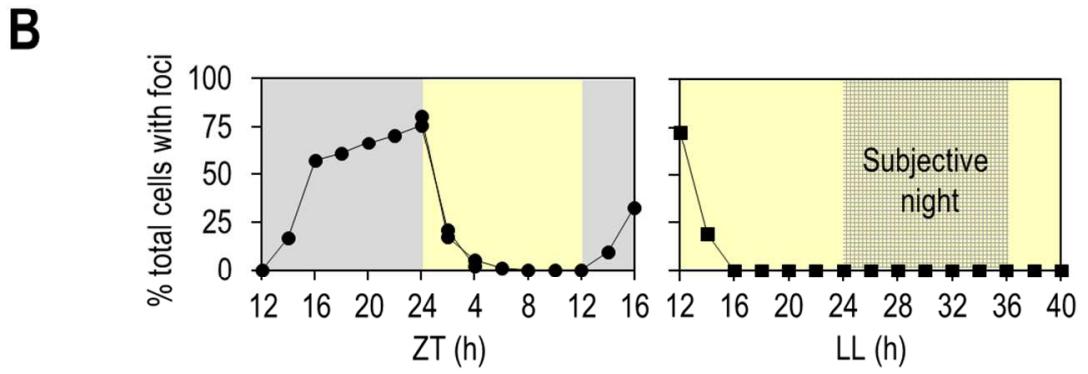

Figure 2: PipX-GFP and PII-YFP localize into foci in darkness. (A) S. elongatus cells were imaged from cultures grown with nitrate in light or dark $(8 \mathrm{~h})$ conditions as indicated. The auto fluorescence (red), PipXGFP (green), PII-YFP (yellow) and ZsG-KaiC (green) signals are shown as indicated. Where appropriated, heatmaps of PipX-GFP or ZsG-KaiC foci, with indication of the numbers detected, show their distribution relative to the cell auto fluorescence border. Colors represent \% of normalized foci present at each cellular position. Histograms to the right show the \% of cells with detectable GFP foci ( 0 to 4$)$ and the number of

cells per category. (B) (Left) Percentage of the cell population that showed PipX-GFP foci in diurnal conditions (light and dark conditions corresponding to yellow and gray regions, respectively). Cells were entrained to opposite light-dark cycles and sampled every 2 hours in either light (ZT 0-12) or dark (ZT 12-

24). (Right) Percentage of the entrained cell population (after two cycles of $12 \mathrm{~h}$ light: $12 \mathrm{~h}$ dark) that showed foci after cells were released into continuous light (LL). The subjective night period is indicated with a grid. ZT (Zeitgeber time) refers to the time relative to "lights on". 


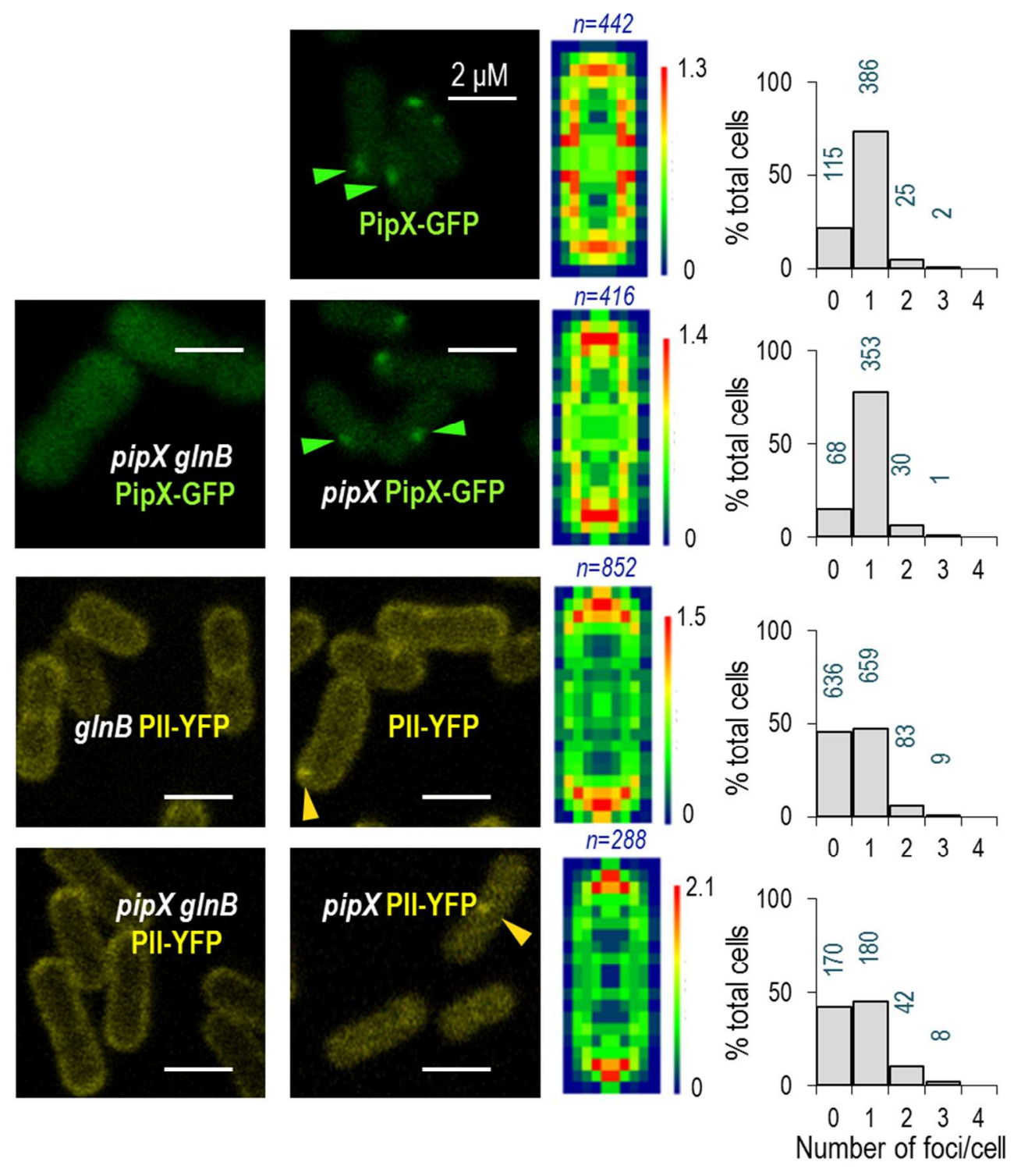

Figure 3: Localization of PipX-GFP or PII-YFP in different genetic backgrounds. S. elongatus cells were imaged from cultures grown with nitrate in dark $(8 \mathrm{~h})$. Heatmaps and histograms correspond to the fociforming strains (pictures from the second column). Examples of detected foci are indicated with arrowheads in the corresponding micrographs. Other details as in Fig. 2.

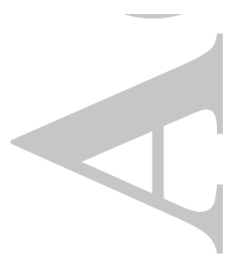




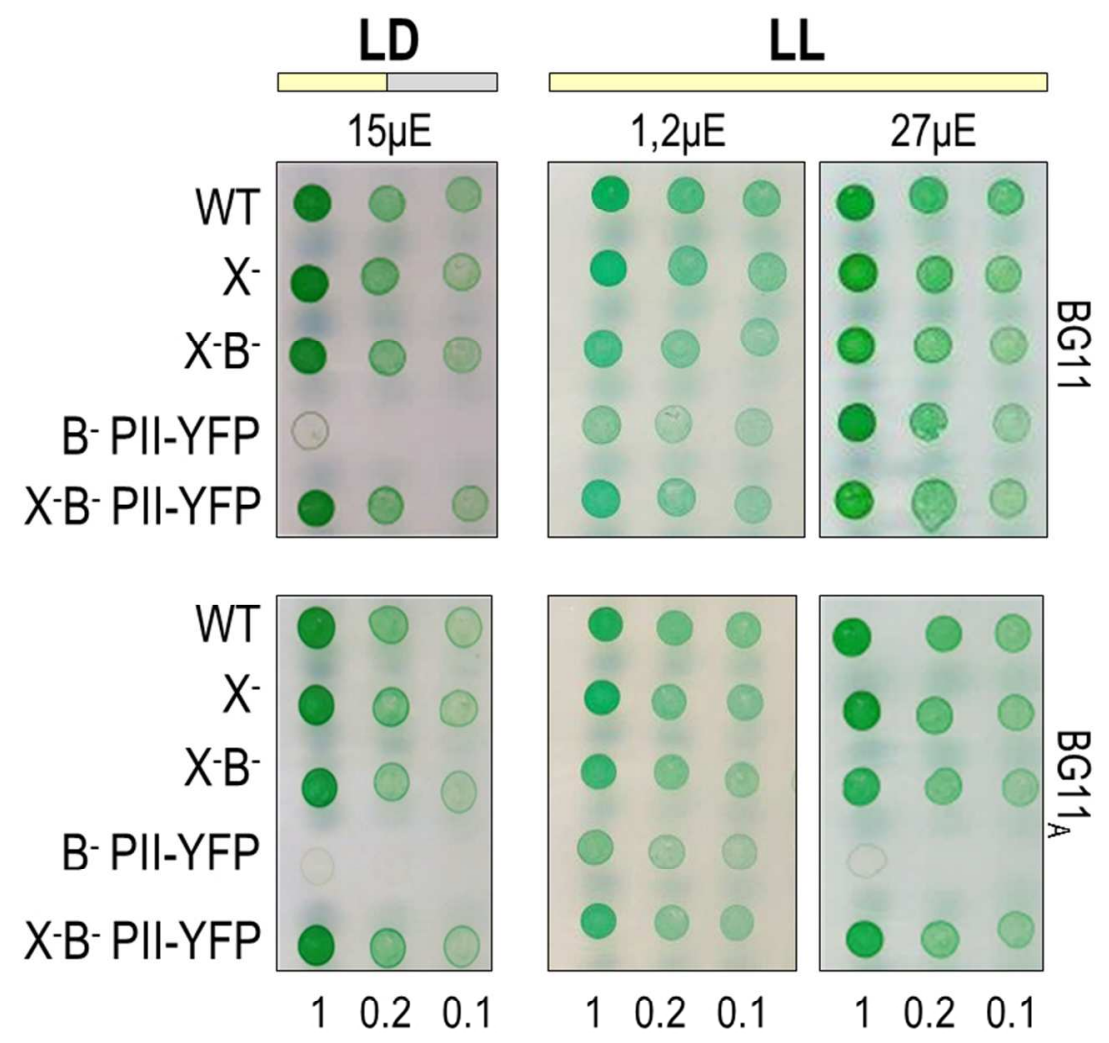

Figure 4: Effects of environmental and genetics backgrounds on $S$. elongatus survival to PipX toxicity. Exponentially growing cultures of WT, pipX (X-), pipXglnB (X-B-), glnB PII-YFP (B- PII-YFP) and pipXglnB PII-YFP (X-B- PII-YFP) strains in constant light (LL) or 12:12 hours light-dark cycles (LD) were adjusted to $0,5\left(\mathrm{OD}_{750}\right)$ prior to drop $5 \mu$ l of the cell suspension onto the plates (BG11 or BG11 $\left.\mathrm{A}\right)$. Numbers on top and bottom refer to light intensities and culture dilutions, respectively. Pictures were taken after 6, 12 and 3 days of incubation (from left to right) in the indicated light conditions. 


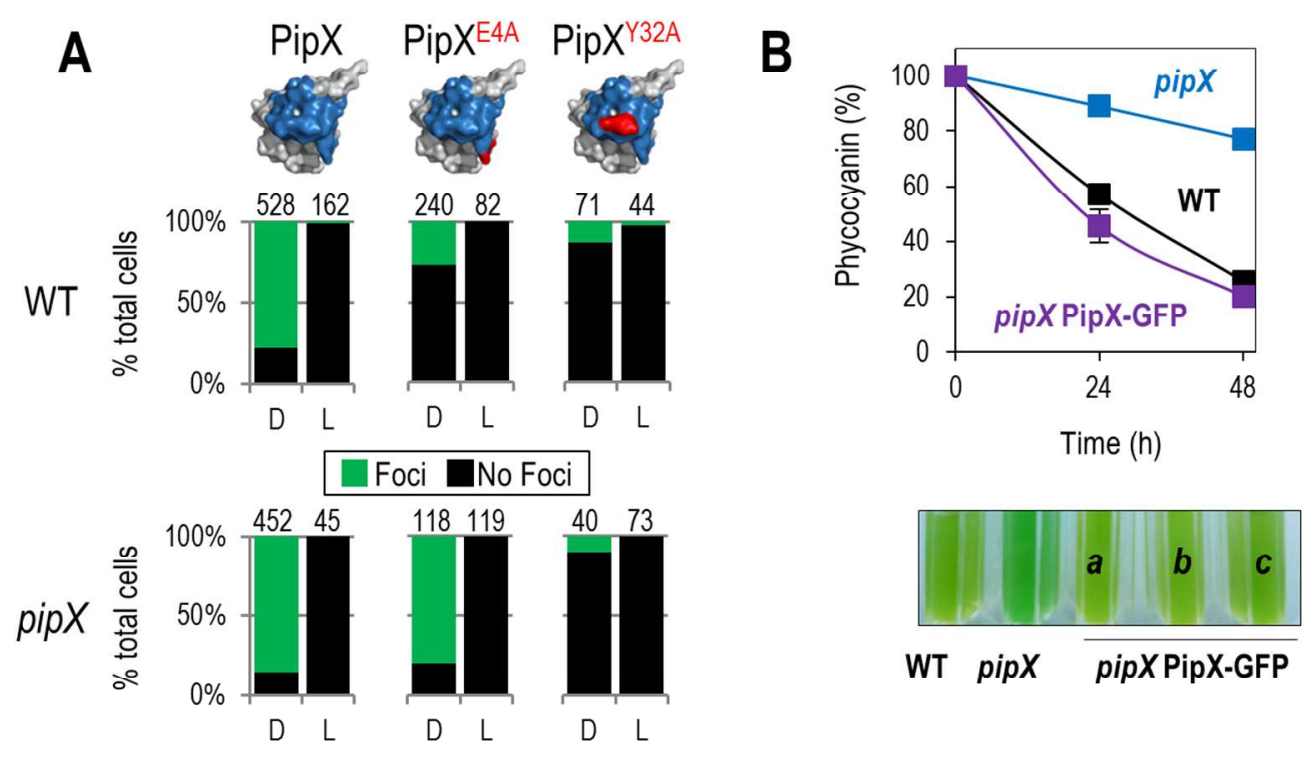

Figure 5: In vivo interactions of PipX-GFP and point mutation derivatives indicate no interference of the GFP tag. (A) Comparative effect of PipX-GFP, PipX $X^{\mathrm{E} 4 \mathrm{~A}}$-GFP and PipX ${ }^{\mathrm{Y} 32 \mathrm{~A}}$-GFP on the number of cells with

fluorescent foci in both WT and pipX strains. PipX structures (accession 2XG8), with the surface interacting with PII in blue and the position of mutated residues in red, illustrate the PipX-GFP derivative present in each strain. The distributions of cells with ( $\geq 1$ focus, green bars) and without foci (black bars) for each strain in light (L) and dark (D) are shown. Total numbers of counted cells are indicated above each bar. (B) PipX-GFP rescues the non-bleaching phenotype of the pipX null mutant. (Top) Relative amount of phycocyanin content after nitrogen deprivation in WT, pipX and pipX PipX-GFP strains. (Bottom) Appearance of the different cultures after nitrogen deprivation (48 hours). The phycocyanin for pipX PipX-GFP corresponds to mean $\pm \mathrm{SD}$ of three independent clones ( $\mathrm{a}, \mathrm{b}$ and $\mathrm{c}$ ). 
A
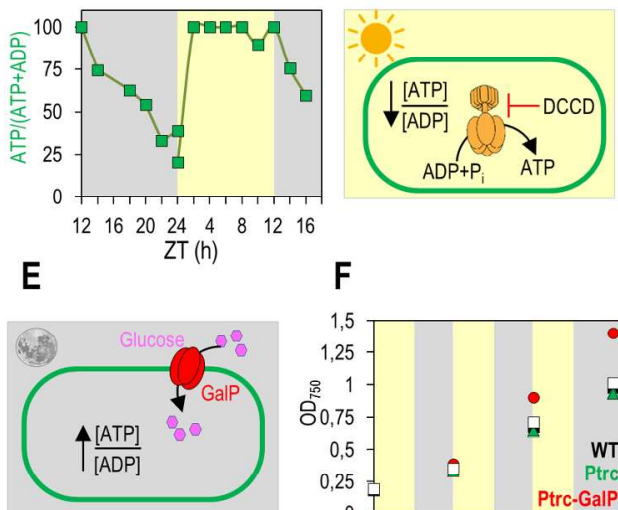

B

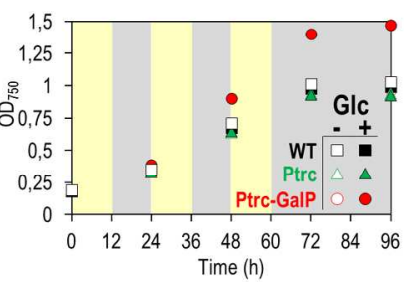

C
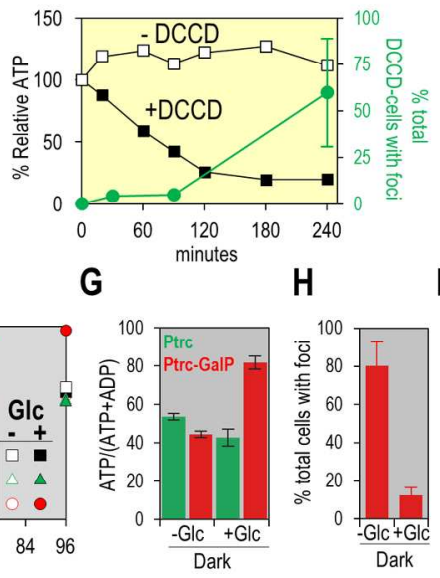

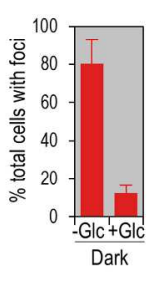

$2 \underline{2 \mu M}+D C C D$ 4h

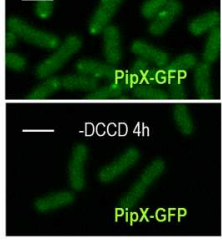

(

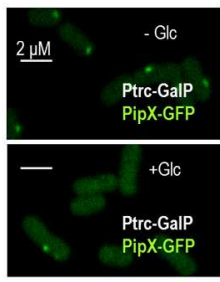

Figure 6: The amount of PipX-GFP /PII-YFP foci inversely correlates with the ATP/ADP ratio. (A) ATP/ADP ratio in $S$. elongatus cells subjected to the light and dark intervals indicated by white and gray regions, respectively. (B) Under light conditions the ATP/ADP ratio decreases upon addition of DCCD, an inhibitor of

F1Fo-ATPase. (C) Percentage of DCCD-treated cells with foci and relative \% of ATP (referred to t0 set to 100 $\%$ ) in light incubated cells (DMSO control) and after addition of DCCD. (D) Representative pictures of cells after 4 hours of treatment with or without DCCD (No foci were detected in the DMSO control). (E) Under dark conditions cells expressing GalP take up Glucose increasing their intracellular ATP/ADP ratio. (F) Growth curve from WT, Ptrc and Ptrc-GalP strains during light and dark conditions (yellow and gray intervals, respectively) with $100 \mu \mathrm{M}$ IPTG and with (filled symbols) or without (open symbols) glucose (Glc) supplementation. (G) ATP/ADP ratio in Ptrc and Ptrc-GalP strains after 10 hours in the dark in medium with or without glucose supplementation. (H) Percentage of cells with PipX-GFP foci in Ptrc-GalP strain after 10 hours of darkness with ( $n=1294$ cells) and without glucose ( $n=2169$ cells). (I) Representative micrographs of cells from $\mathrm{H}$. 